\title{
Biosynthesis of TDP-L-Mycarose: The Specificity of a Single Enzyme Governs the Outcome of the Pathway
}

\author{
Haruoko Takahashi, Yung-nan Liu, Huawei Chen, and Hung-wen Liu* \\ Division of Medicinal Chemistry, College of Pharmacy and Department of Chemistry and \\ Biochemistry, University of Texas at Austin, Austin, Texas 78712
}

\section{Supporting Information}

Spectral data of 6: ${ }^{1} \mathrm{H}$ NMR $\left(500 \mathrm{MHz},{ }^{2} \mathrm{H}_{2} \mathrm{O}\right)$ of $6: \delta 1.11(3 \mathrm{H}, \mathrm{d}, J=6.0 \mathrm{~Hz}, 5-\mathrm{Me}$ hydrated form), 1.15 (3H, d, $J=6.0 \mathrm{~Hz}, 5-\mathrm{Me}$ keto form), 1.37 (3H, s, 3-Me hydrated form), 1.45 (3H, s, 3-Me keto form), 1.82 (3H, s, 5"-Me), 1.92 (2H, m, 2-Hs hydrated form), 2.23-2.32 (2H, m, 2'Hs), 2.40 (2H, m, 2-Hs keto form), 4.01-4.10 (3H, m, 5-H hydrate form, 4'-H, 5'-Hs), 4.47-4.53 (1H, m, 3'-H), $4.72(1 \mathrm{H}, \mathrm{q}, J=6.0 \mathrm{~Hz}, 5-\mathrm{H}$ keto form), 5.49 (1H, m, 1-H hydrated form), 5.64 (1H, m, 1-H keto form), $6.24\left(1 \mathrm{H}, \mathrm{t}, J=4.2 \mathrm{~Hz}, 1\right.$ '-H), $7.61(1 \mathrm{H}, \mathrm{s}, 6 "-\mathrm{H}) .{ }^{13} \mathrm{C}$ NMR $(75 \mathrm{MHz}$, ${ }^{2} \mathrm{H}_{2} \mathrm{O}$, hydrated form) $\delta 11.6,12.0,23.5,38.3,40.9(\mathrm{~d}, J(\mathrm{C}, \mathrm{P})=7.5 \mathrm{~Hz} ; \mathrm{C}-2), 65.3\left(\mathrm{~d},{ }^{2} J(\mathrm{C}, \mathrm{P})=\right.$ 5.6 Hz; C-5'), 68.1, 70.7, 71.8, 84.7, $85.0\left(\mathrm{~d},{ }^{3} J(\mathrm{C}, \mathrm{P})=7.5 \mathrm{~Hz}\right.$; C-4'), $94.0\left(\mathrm{~d},{ }^{2} J(\mathrm{C}, \mathrm{P})=5.3 \mathrm{~Hz}\right.$; C-1), 94.3, 111.6, 137.2, 151.6, 166.5. The ratio between the hydrated form and the keto form is approximately $3: 1$.

Spectral data of 8: ${ }^{1} \mathrm{H}$ NMR $\left(500 \mathrm{MHz}, \mathrm{D}_{2} \mathrm{O}\right) \delta 1.14(3 \mathrm{H}, \mathrm{s}, 3-\mathrm{Me}), 1.16(3 \mathrm{H}, \mathrm{d}, J=6.4 \mathrm{~Hz}, 5-$ Me), $1.56\left(1 \mathrm{H}, \mathrm{dd}, J=9.8,14.0 \mathrm{~Hz}, 2-\mathrm{H}_{\mathrm{ax}}\right), 1.82(3 \mathrm{H}, \mathrm{br} \mathrm{s}, 5 "-\mathrm{Me}), 2.04(1 \mathrm{H}, \mathrm{dd}, J=2.2,14.0$ $\left.\mathrm{Hz}, 2-\mathrm{H}_{\mathrm{eq}}\right), 2.25\left(2 \mathrm{H}, \mathrm{m}, 2^{\prime}-\mathrm{H}\right), 2.97(1 \mathrm{H}, \mathrm{d}, J=9.8 \mathrm{~Hz}, 4-\mathrm{H}), 3.70(1 \mathrm{H}, \mathrm{dq}, J=9.8,6.4 \mathrm{~Hz}, 5-\mathrm{H})$, $4.06\left(3 \mathrm{H}, \mathrm{m}, 4^{\prime}-\right.$ and 5'-H), $4.47\left(1 \mathrm{H}, \mathrm{m}, 3^{\prime}-\mathrm{H}\right), 5.33(1 \mathrm{H}, \mathrm{ddd}, J=2.2,7.8,9.8 \mathrm{~Hz}, 1-\mathrm{H}), 6.25$ $(1 \mathrm{H}, \mathrm{m}, 1 \mathrm{\prime}-\mathrm{H}), 7.64(1 \mathrm{H}$, br s, 6"-H). Compound $\mathbf{8}$ is relatively labile; it is readily hydrolyzed to release the TDP part. Spectral data for the free sugar of 8: ${ }^{1} \mathrm{H}$ NMR $\left(500 \mathrm{MHz}, \mathrm{D}_{2} \mathrm{O}\right) \delta 1.18(3 \mathrm{H}$, 
$\mathrm{d}, J=6.3 \mathrm{~Hz}, 5-\mathrm{Me}), 1.19(3 \mathrm{H}, \mathrm{s}, 3-\mathrm{Me}), 1.52\left(1 \mathrm{H}, \mathrm{dd}, J=10.0,13.9 \mathrm{~Hz}, 2-\mathrm{H}_{\mathrm{ax}}\right), 1.92(1 \mathrm{H}, \mathrm{dd}, J$ $\left.=2.1,13.9 \mathrm{~Hz}, 2-\mathrm{H}_{\mathrm{eq}}\right), 3.01(1 \mathrm{H}, \mathrm{d}, J=9.7 \mathrm{~Hz}, 4-\mathrm{H}), 3.67(1 \mathrm{H}, \mathrm{dq}, J=9.7,6.3 \mathrm{~Hz}, 5-\mathrm{H}), 4.99$ $(1 \mathrm{H}, \mathrm{dd}, J=2.1,10.0 \mathrm{~Hz}, 1-\mathrm{H}) ;{ }^{13} \mathrm{C}$ NMR $\left(125 \mathrm{MHz}, \mathrm{D}_{2} \mathrm{O}\right) \delta 17.6$ (5-Me), 25.9 (3-Me), 44.3 (C2), 70.6 (C-5), 71.2 (C-3), 76.2 (C-4), 92.2 (C-1); high-resolution CI-MS calcd for $\mathrm{C}_{7} \mathrm{H}_{15} \mathrm{O}_{4}(\mathrm{M}+$ $\mathrm{H})^{+}$163.0970, found 163.0973.

Spectral data of 9: ${ }^{1} \mathrm{H}$ NMR $\left(500 \mathrm{MHz}, \mathrm{D}_{2} \mathrm{O}\right) \delta 1.14(3 \mathrm{H}, \mathrm{d}, J=6.6 \mathrm{~Hz}, 5-\mathrm{Me}), 1.36(3 \mathrm{H}, \mathrm{s}$, 3-Me), $1.80\left(1 \mathrm{H}, \mathrm{d}, J=14.0 \mathrm{~Hz}, 2-\mathrm{H}_{\mathrm{ax}}\right), 1.85\left(3 \mathrm{H}\right.$, br s, 5"-Me), $1.88\left(1 \mathrm{H}, \mathrm{m}, 2-\mathrm{H}_{\mathrm{eq}}\right), 2.29(2 \mathrm{H}, \mathrm{m}$, 2'-H), $3.25(1 \mathrm{H}, \mathrm{s}, 4-\mathrm{H}), 4.09$ (3H, m, 4'- and 5'-H), $4.19(1 \mathrm{H}, \mathrm{q}, J=6.6 \mathrm{~Hz}, 5-\mathrm{H}), 4.55(1 \mathrm{H}, \mathrm{m}$, 3'-H), $5.56(1 \mathrm{H}, \mathrm{m}, 1-\mathrm{H}), 6.26\left(1 \mathrm{H}, \mathrm{t}, J=7.0 \mathrm{~Hz}, 1^{\prime}-\mathrm{H}\right), 7.67(1 \mathrm{H}, \mathrm{m}, 6 "-\mathrm{H})$. Compound 9 is relatively labile; it is readily hydrolyzed to release the TDP part. Spectral data of the free sugar of 9: ${ }^{1} \mathrm{H}$ NMR (500 MHz, $\left.\mathrm{D}_{2} \mathrm{O}\right) \delta 1.16(3 \mathrm{H}, \mathrm{d}, J=6.5 \mathrm{~Hz}, 5-\mathrm{Me}), 1.21(3 \mathrm{H}, \mathrm{s}, 3-\mathrm{Me}), 1.59(1 \mathrm{H}$, br t, $\left.J=12 \mathrm{~Hz}, 2-\mathrm{H}_{\text {ax }}\right), 1.71\left(1 \mathrm{H}, \mathrm{ddd}, J=1.1,2.4,12.4 \mathrm{~Hz}, 2-\mathrm{H}_{\text {eq }}\right), 3.15(1 \mathrm{H}$, br s, 4-H $), 3.76$ $(1 \mathrm{H}, \mathrm{dq}, J=1.1,6.5 \mathrm{~Hz}, 5-\mathrm{H}), 4.77(1 \mathrm{H}, \mathrm{dd}, J=2.4,10.0 \mathrm{~Hz}, 1-\mathrm{H}) ;{ }^{13} \mathrm{C} \mathrm{NMR}\left(125 \mathrm{MHz}, \mathrm{D}_{2} \mathrm{O}\right) \delta$ 16.5 (5-Me), 23.5 (3-Me), 40.5 (C-2), 70.2 (C-5), 71.3 (C-3), 73.7 (C-4), 93.3 (C-1); highresolution CI-MS calcd for $\mathrm{C}_{7} \mathrm{H}_{15} \mathrm{O}_{4}(\mathrm{M}+\mathrm{H})^{+}$163.0970, found 163.0975. This sugar 9 has not been found in natural products.

Spectral data of 11: ${ }^{1} \mathrm{H}$ NMR $\left(500 \mathrm{MHz}, \mathrm{D}_{2} \mathrm{O}\right) \delta 1.19(3 \mathrm{H}, \mathrm{d}, J=6.2 \mathrm{~Hz}, 5-\mathrm{Me}), 1.49(1 \mathrm{H}, \mathrm{dt}$, $\left.J=10.0,11.8 \mathrm{~Hz}, 2-\mathrm{H}_{\mathrm{ax}}\right), 1.81\left(3 \mathrm{H}\right.$, br s, 5"-Me), $2.25\left(2 \mathrm{H}, \mathrm{m}, 2^{\prime}-\mathrm{H}\right), 2.28\left(1 \mathrm{H}, \mathrm{m}, 2-\mathrm{H}_{\mathrm{eq}}\right), 2.94$ $(1 \mathrm{H}, \mathrm{t}, J=9.4 \mathrm{~Hz}, 4-\mathrm{H}), 3.34(1 \mathrm{H}, \mathrm{dq}, J=9.4,6.2 \mathrm{~Hz}, 5-\mathrm{H}), 3.57(1 \mathrm{H}, \mathrm{ddd}, J=5.1,9.4,11.8 \mathrm{~Hz}$, 3-H), 4.05 (3H, m, 4'- and 5'-H), 4.53 (1H, m, 3'-H), $5.15(1 \mathrm{H}, \mathrm{ddd}, J=2.0,8.0,10.0 \mathrm{~Hz}, 1-\mathrm{H})$, $6.23\left(1 \mathrm{H}, \mathrm{t}, J=6.9 \mathrm{~Hz}, 1^{\prime}-\mathrm{H}\right), 7.65(1 \mathrm{H}, \mathrm{br} \mathrm{s}, 6 "-\mathrm{H})$. Compound 11 is relatively labile; it is readily hydrolyzed to release the TDP part. Spectral data for the free sugar of 11: ${ }^{1} \mathrm{H}$ NMR $(500$ $\left.\mathrm{MHz}, \mathrm{D}_{2} \mathrm{O}\right) \delta 1.19(3 \mathrm{H}, \mathrm{d}, J=6.2 \mathrm{~Hz}, 5-\mathrm{Me}), 1.42\left(1 \mathrm{H}, \mathrm{dt}, J=9.8,11.6 \mathrm{~Hz}, 2-\mathrm{H}_{\mathrm{ax}}\right), 2.16(1 \mathrm{H}$, 
ddd, $\left.J=2.2,5.0,11.6 \mathrm{~Hz}, 2-\mathrm{H}_{\mathrm{eq}}\right), 2.96(1 \mathrm{H}, \mathrm{t}, J=9.2 \mathrm{~Hz}, 4-\mathrm{H}), 3.32(1 \mathrm{H}, \mathrm{dq}, J=9.2,6.2 \mathrm{~Hz}, 5-$ H), $3.57(1 \mathrm{H}, \mathrm{ddd}, J=5.0,9.2,11.6 \mathrm{~Hz}, 3-\mathrm{H}), 4.82(1 \mathrm{H}, \mathrm{dd}, J=2.2,9.8 \mathrm{~Hz}, 1-\mathrm{H}) ;{ }^{13} \mathrm{C}$ NMR (125 MHz, $\left.\mathrm{D}_{2} \mathrm{O}\right)$ ठ 17.1 (5-Me), 40.0 (C-2), 70.4 (C-3), 72.1 (C-5), 76.5 (C-4), 93.4 (C-1); highresolution CI-MS calcd for $\mathrm{C}_{6} \mathrm{H}_{13} \mathrm{O}_{4}(\mathrm{M}+\mathrm{H})^{+}$149.0814, found 149.0820. The sugar part of compound $\mathbf{1 1}$ has not been found in natural products. Its 3-O-methylated derivative is known as L-oleandrose, which is present in avermectins and notonesomycin A. 